\title{
Estimation de la dose efficace au poste de travail : intérêt de disposer d'un moyen de mesure adapté
}

\author{
S. MÉNARD ${ }^{1,2}$, C. FURSTOSS ${ }^{1}$, L. LACHEVRE ${ }^{1}$
}

(Manuscrit reçu le 14 mai 2007, accepté le 10 novembre 2007)

RÉSUMÉ Les valeurs limites annuelles d'exposition de l'organisme entier des travailleurs et du public sont définies à l'aide de la dose efficace $\boldsymbol{E}$. Les dosimètres individuels portés par les travailleurs évaluent un estimateur de cette grandeur de protection : l'équivalent de dose individuel. Cet article présente les deux méthodes de calcul des équivalents de dose et de la dose efficace ainsi que les résultats obtenus dans un champ mixte neutronique-photonique auprès d'une boîte à gants. Les équivalents de dose calculés pour ce poste de travail dépendent des hypothèses de calcul. Estimer la dose efficace à partir de ces résultats se révèle de ce fait délicat. Cette étude démontre ainsi l'intérêt de disposer d'un moyen de mesure de la dose efficace.

ABSTRACT Interest to have a suitable instrument for the effective dose estimation at workplaces.

The annual limit values of whole body exposure for workers and public whole are defined using the effective dose $E$. The personal dosimeters worn by workers give an estimation of this effective dose: the personal dose equivalent. This paper presents the two calculation methods of dose equivalents and effective dose and the results obtained in a mixed neutron-photon field nearby a glove box. The dose equivalents calculated for this workplace depend on the calculation hypothesis. Then the estimation of the effective dose using these results turns out to be delicate. This study shows the interest to develop an effective dose measurement instrument.

Keywords: effective dose / dose equivalent / MCNPX / conversion coefficient / neutron

Le décret $\mathrm{n}^{\circ}$ 2003-296 du 31 mars 2003, relatif à la protection des travailleurs contre les dangers des rayonnements ionisants, établit à l'article R. 232-116 du code de travail que la somme des doses efficaces reçues par exposition externe et interne ne doit pas dépasser $20 \mathrm{mSv}$ sur douze mois consécutifs. La méthode de calcul de la dose efficace, objet d'un arrêté d'application de ce décret, se base sur la définition de la publication 60 de la Commission internationale de protection radiologique (ICRP, 1990).

Les valeurs limites d'exposition de l'organisme entier et les valeurs de référence pour la délimitation des installations sont définies par rapport à la dose efficace,

${ }^{1}$ IRSN, Direction de la Radioprotection de l'Homme, B.P. 17, 92262 Fontenay-aux-Roses Cedex, France.

${ }^{2}$ Adresse actuelle de S. Ménard : CEA, Centre DAM-Ile de France, Bruyères-le-Châtel, 91297 Arpajon Cedex, France. 
grandeur de protection, qui n'est pas directement mesurable. Les instruments de mesure individuels et d'ambiance évaluent des estimateurs de la dose efficace $E$ : les équivalents de dose $H_{p}(10)$ et $H^{*}(10)$ introduits avec cet objectif par la Commission internationale des unités radiologiques (CIUR) dans son rapport 57 (ICRU, 1998). Ce document présente leurs limites en fonction du type, de l'énergie et de l'angle du rayonnement incident. À ces limites théoriques se cumulent les sous- et surestimations expérimentales des équivalents de dose évalués par les instruments de mesure d'ambiance et les dosimètres individuels.

L'écart sur l'estimation de la dose efficace à l'aide des grandeurs opérationnelles dépend donc des conditions d'exposition aux rayonnements du travailleur à son poste. Cet écart peut cependant être estimé par le calcul mais est difficilement quantifiable par la mesure faute de disposer d'instruments évaluant les distributions en énergie et en angle de la fluence.

\section{Méthode de calcul des grandeurs de radioprotection}

La dose efficace et les équivalents de dose peuvent être calculés selon deux méthodes numériques :

- à partir de leur définition en utilisant des fantômes adaptés à la grandeur (fantômes anthropomorphe, parallélépipédique et sphériques),

- à partir des distributions en angle et en énergie de la fluence pondérées par les coefficients de conversion publiés dans le rapport 57 de la CIUR.

\subsection{Première méthode}

La première méthode de calcul des grandeurs de protection et opérationnelles consiste à utiliser un code Monte Carlo de transport de rayonnements et des fantômes adaptés à la grandeur ciblée :

- un fantôme anthropomorphe pour calculer la dose efficace $E$,

- une sphère de $15 \mathrm{~cm}$ de rayon en équivalent-tissu CIUR pour calculer l'équivalent de dose ambiant $H^{*}(10)$,

- un parallélépipède de $30 \times 30 \times 15 \mathrm{~cm}$ en équivalent-tissu CIUR pour évaluer l'équivalent de dose individuel $H_{p}(10)$.

Les valeurs de dose efficace publiées dans le rapport 57 de la CIUR pour les neutrons ont été estimées en utilisant quatre codes Monte Carlo de transport et cinq modèles mathématiques permettant de simuler le corps humain et les organes/tissus radiosensibles intervenant dans le calcul de la dose efficace $E$.

Le code MCNPX 2.5.0 (Hendricks et al., 2005) est le code Monte Carlo de transport utilisé par le service de dosimétrie de l'IRSN pour les évaluations 
numériques de la dose dans le cas d'exposition externe en situation normale ou accidentelle. Un modèle de fantôme mathématique hermaphrodite anthropomorphe, basé sur les spécifications de Cristy et Eckerman auxquelles des modifications (volumes, thyroïde) ont été apportées (Cristy et Eckerman, 1987; Furstoss et Ménard 2005), ainsi que les fantômes sexués ADAM et EVA (Kramer et al., 1982) ont été modélisés au format du code MCNPX.

La dose efficace est définie par l'équation :

$$
E=\sum_{T} w_{T} \sum_{R} w_{R} D_{T, R}
$$

où $w_{T}$ est le facteur de pondération du tissu irradié, $w_{R}$ est le facteur de pondération du rayonnement incident et $D_{T, R}$ est la dose absorbée moyenne à l'organe ou tissu $T$.

La publication 60 de la CIPR et le rapport 57 de la CIUR définissent :

- les 13 tissus/organes radiosensibles et la composition du reste intervenant dans le calcul de la dose efficace,

- les valeurs du facteur de pondération $w_{T}$,

- la variation du facteur de pondération $w_{R}$ en fonction du type de rayonnement incident sur le fantôme anthropomorphe et de l'énergie des neutrons incidents.

Par ailleurs, les hypothèses de calcul retenues par les auteurs des simulations sont également présentées. La première hypothèse est la validité de l'approximation kerma, qui suppose que les particules chargées déposent leur énergie sur place, pour les neutrons d'énergie inférieure à $20 \mathrm{MeV}$ (ICRU, 1998, p. 17). La seconde hypothèse concerne le calcul de la dose au reste: les auteurs ont retenu une moyenne arithmétique des doses absorbées des 10 organes/tissus du reste (ICRU, 1998, p. 40).

Seule la première hypothèse a été retenue pour nos calculs. La dose au reste a été calculée en tenant compte de la masse de chacun des 10 organes/tissus.

La principale difficulté dans l'évaluation de $E$ réside dans la dépendance en énergie du facteur de pondération $w_{R}$ qui est appliqué sur les doses dues aux neutrons et à ses rayonnements secondaires produits dans le fantôme. La publication 60 de la CIPR recommande de discrétiser le calcul de $E$ en tranches d'énergie neutron en s'appuyant soit sur les bornes en énergie des différentes valeurs du $w_{R}$ ( $c f$. Tab. A-2 de la CIPR) soit sur l'approximation de la fonction continue $w_{R}\left(E_{n}\right)$ des neutrons publiee dans la publication 60 de la CIPR. De plus, il faut distinguer des doses dues à des photons produits par des neutrons des doses dues à des photons incidents sur le fantôme qui ont diffusé. 
De ce fait, l'évaluation de la dose efficace $E$ requiert de disposer des informations sur :

- la composition du champ incident en photons/neutrons,

- la distribution en énergie de la fluence neutronique irradiant le fantôme,

- les distributions spatiales et angulaires des rayonnements incidents.

La programmation au niveau de MCNPX consiste :

- à ne transporter que les photons et neutrons en considérant que toutes les particules chargées déposent leur énergie au point d'interaction (approximation kerma),

- à encaisser les dépôts d'énergie, «tallies f6 », du code pour chacun des organes/tissus radiosensibles,

- à séparer les calculs en fonction des rayonnements incidents et de leur énergie, ceci pour appliquer le bon facteur de pondération aux doses absorbées calculées.

Cela implique de connaître avec précision la source de rayonnements incidents, c'est-à-dire la source directe et ses diffusés dans l'environnement d'ambiance du travailleur.

Les équivalents de dose ambiant $H^{*}(10)$ et $H_{p}(10)$ sont calculés à $1 \mathrm{~cm}$ de profondeur dans les fantômes CIUR à l'aide de l'équation de l'équivalent de dose $H$ :

$$
H=\int Q(L) \frac{d D(L)}{d L} d L
$$

où $L$ est le transfert d'énergie linéique, $Q(L)$ est le facteur de qualité du rayonnement et $d D$ est la distribution de la dose absorbée en un point de particules chargées de transfert d'énergie linéique compris entre $L$ et $L+d L$.

Le facteur de qualité $Q(L)$ des particules chargées produites par les rayonnements photoniques est supposé égal à 1 quelque soit $L$ pour les calculs de $H^{*}(10)$ et $H_{p}(10)$. Le facteur de qualité $Q(L)$ affecté aux particules chargées produites par les neutrons dépend fortement de $L$. Par conséquent, le calcul des équivalents de dose requiert de connaître le transfert linéique d'énergie $L$ au point de calcul de la dose ; ce qui n'est pas possible avec MCNPX. C'est pourquoi pour les neutrons, les facteurs de qualité pondérés $Q_{n} k_{f}$ de Siebert et Schumacher (1992) sont utilisés en association avec le calcul de la fluence en un point à $1 \mathrm{~cm}$ de profondeur dans le fantôme CIUR pour évaluer la contribution neutron de l'équivalent de dose. La contribution des photons créés par l'interaction des neutrons dans le fantôme est déduite de la dose en un point puisque $Q(L)=1$ pour ces rayonnements. 
Les simulations MCNPX ont consisté à calculer :

- les encaissements des distributions spectrales en énergie de la fluence « tallies f4 » pondérées par les coefficients neutroniques $Q_{n} k_{f}$ tabulés (carte df) en fonction des énergies neutroniques (carte de) pour estimer la contribution neutronique,

- les encaissements de dépôts d'énergie en un point «tallies f6 » pour la composante photonique.

\subsection{Seconde méthode}

La détermination des grandeurs peut être effectuée à l'aide des coefficients de conversion publiés dans le rapport 57 de la CIUR. C'est la méthode la plus couramment utilisée. Des codes déterministes ou Monte Carlo peuvent être employés. Cependant, la distribution en angle de la fluence n'étant pas calculable par les codes déterministes, leur utilisation requiert quelques précautions. Les distributions spectrales des fluences obtenues avec les encaissements, «tallies f4 et f5 », de MCNPX peuvent ainsi être utilisés en association avec les cartes de tabulation des coefficients en fonction de l'énergie de et df.

\section{Validation du schéma de calcul de la dose efficace $\mathbf{E}$}

La complexité du calcul de la dose efficace $E$ pour les neutrons nécessite dans un premier temps de valider le schéma de calcul en comparant les résultats obtenus à ceux de la littérature de la CIPR et de la CIUR.

Trois géométries de fantômes anthropomorphes (ADAM, EVA et celui de l'IRSN) ont donc été modélisées pour les configurations d'exposition neutronique suivantes :

- antéropostérieure AP : irradiation de face du fantôme,

- latérale gauche LLAT et droite RLAT,

- postéro-antérieure PA : irradiation de dos du fantôme.

Les distributions des neutrons sont mononénergétiques et unidirectionnelles. De plus, le fantôme est uniformément irradié.

Les figures 1 et 2 présentent les résultats obtenus pour des irradiations neutroniques monoénergétiques en géométrie AP et PA.

Aussi bien en AP qu'en PA et en LLAT, les différences géométriques entre les fantômes conduisent à des écarts sur l'évaluation de la dose efficace. La comparaison aux valeurs de coefficients de conversion dose efficace-fluence publiés (ICRP, 1990; ICRU, 1998) est présentée sur les figures 3 et 4. Les 


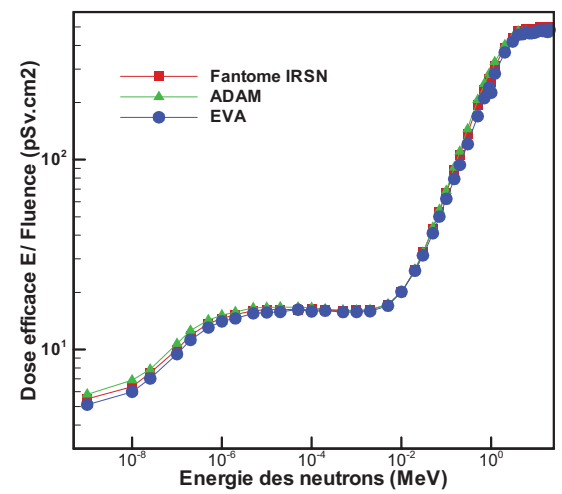

Figure 1 - Coefficients de conversion en AP. Conversion coefficients in AP.

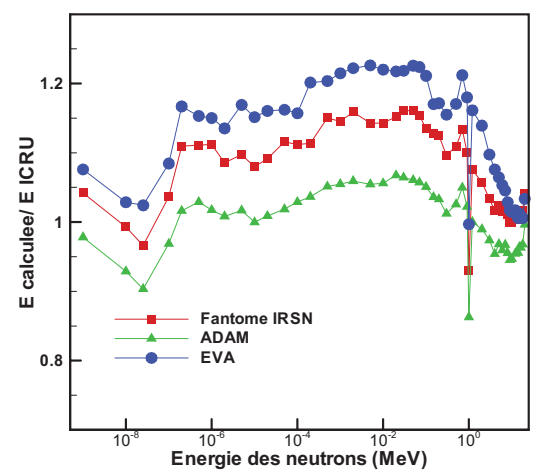

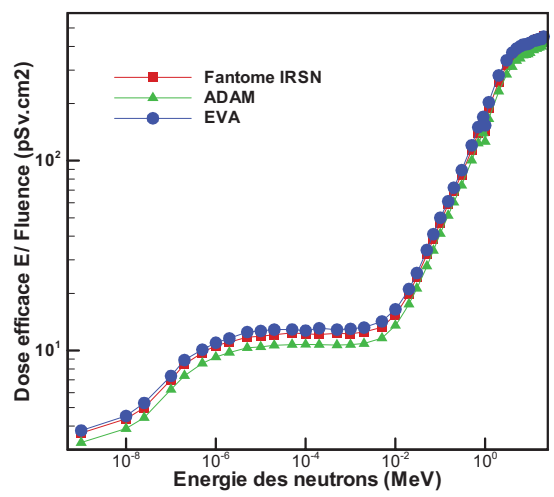

Figure 2 - Coefficients de conversion en PA. Conversion coefficients in PA.

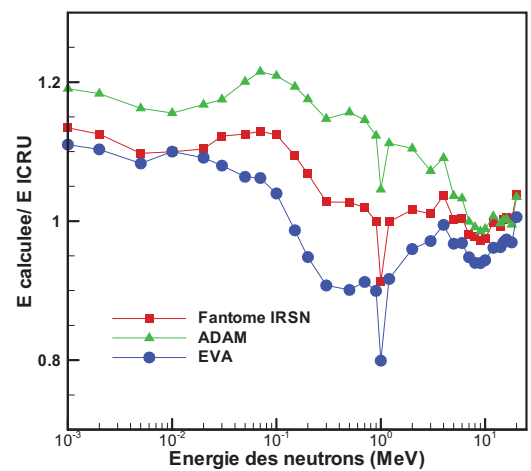

Figure 3 - Rapports des doses efficaces calculées dans ce travail sur celles publiées dans (ICRU, 1998) : géométrie AP (droite), géométrie PA (gauche).

The ratios of effective doses calculated in this work to those published in (ICRU, 1998): AP geometry (right), PA geometry (left).

coefficients de conversion dose efficace-fluence de la CIUR sont le résultat des calculs de 7 groupes, ayant utilisé soit des codes soit des fantômes différents, et qui ont interpolés par des splines de moindres carrés leurs calculs de doses absorbées moyennes aux organes. Les écarts constatés entre les trois fantômes ADAM, EVA et IRSN sont du même ordre de grandeur que ceux constatés par les membres des 7 groupes. Le fantôme retenu par l'IRSN étant hermaphrodite, des écarts, inférieurs à $10 \%$, sur la dose efficace $E$ par rapport aux valeurs interpolées de la CIUR sont constatés. Étudier l'origine des écarts nécessite de comparer les doses absorbées moyennes évaluées pour chacun des organes/tissus. Une telle comparaison a été réalisée pour quelques énergies et des conditions d'irradiation différentes. La 

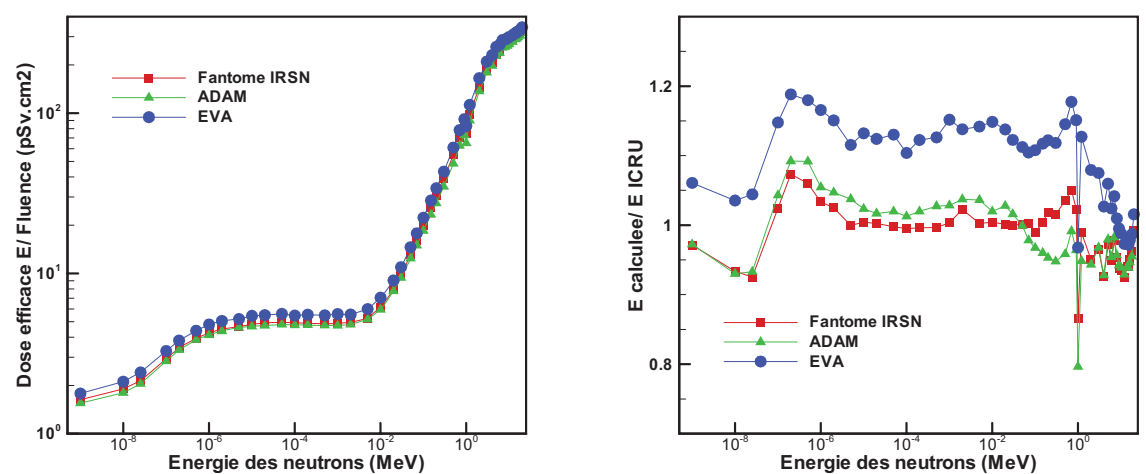

Figure 4 - Calculs de dose efficace en irradiation LLAT (gauche) et normalisés à ceux de l'ICRU (droite). Calculations of effective doses in LAT geometry irradiation (left), normalised to ICRU results (right).

conclusion est identique à celles des 7 groupes ayant réalisé les calculs neutrons de la CIUR 57. Les différences sont principalement dues en l'occurrence à des différences géométriques entre les trois modèles. De plus, les interpolations sur les doses absorbées moyennes aux organes s'ajoutent à ces différences et expliquent les écarts avec les doses efficaces de la CIUR. La fonction continue $w_{R}\left(E_{n}\right)$ est appliquée sur les doses calculées. Le décrochement systématique des doses efficaces à $1 \mathrm{MeV}$ quel que soit l'irradiation résulte d'un artefact de calcul; la valeur maximum des facteurs de pondération évaluées avec la fonction $w_{R}(E)$ (Éq. (2.5), p. 7, ICRU, 1998) est obtenue à 0,9 MeV. Les doses absorbées moyennes aux organes calculées pour ces deux énergies ne compensent pas les variations du facteur de pondération. Aucun décrochement n'est observé pour les résultats publiés dans (ICRU, 1998). On peut supposer que cela est dû aux interpolations réalisées.

$\mathrm{Au}$ vu des résultats, nous pouvons considérer que le fantôme mathématique anthropomorphe est validé pour calculer les doses efficaces pour les neutrons. D'autre part, les travaux de Furstoss ont montré que l'utilisation de ce fantôme et du code MCNPX conduit à un écart de $5 \%$ en champs photoniques (Furstoss, 2006; Furstoss et al., 2007).

\section{Calcul des grandeurs pour un champ représentatif d'un poste de travail}

Les champs de rayonnements auxquels sont exposés les travailleurs à leurs postes diffèrent généralement des irradiations AP, PA, LAT de la CIUR. Les distributions angulaires et énergétiques de la fluence ne sont pas homogènes, la plupart du temps, sur toute la hauteur du fantôme anthropomorphe. 


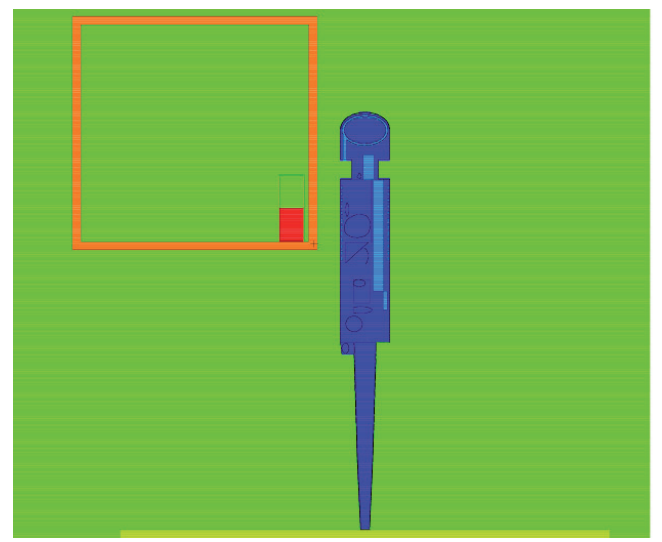

Figure 5-Vue schématique du fantôme IRSN et de la boîte à gants.

Schematic view of the IRSN phantom and the glove box.

L'évaluation de la dose efficace $E$ et de la grandeur opérationnelle $H_{p}(10)$ à partir de la seconde méthode, c'est-à-dire à l'aide des coefficients de conversion, suppose de calculer la fluence. Le choix des points de calcul de la fluence peut conduire à des écarts importants lorsque les distributions des rayonnements incidents sur l'Homme exposé sont hétérogènes.

Le calcul des grandeurs à l'aide de la première méthode se révèle tout aussi complexe dans le cas des champs hétérogènes car le positionnement des fantômes peut induire des variations importantes sur la valeur calculée et sur la bonne évaluation de la dose efficace $E$ à l'aide de $H_{p}(10)$.

L'évaluation dosimétrique de l'exposition d'un travailleur auprès d'une boîte à gants permet d'illustrer la complexité du calcul.

\section{4. Évaluation dosimétrique auprès d'une boîte à gants}

La figure 5 présente une vue schématique du poste de travail étudié. La boîte à gants est en verre au plomb de 3,6 cm d'épaisseur et de dimensions $200 \times 100 \times$ $100 \mathrm{~cm}$ et le fond de la boîte est à $140 \mathrm{~cm}$ du sol. L'évaluation dosimétrique est réalisée pour les neutrons de fission spontanée issus de la source (oxyde de plutonium) contenue dans le pot.

Le spectre d'émission des neutrons de fission spontanée est un spectre de Watt entre 0 et $14 \mathrm{MeV}$ et des paramètres $(a=0,965, b=2,29)$.

\section{1. Évaluation des grandeurs à partir de leur définition (1 ${ }^{\text {re }}$ méthode)}

La dose efficace $E$ et les équivalents de dose ambiants et individuels de ce poste de travail ont été évalués avec MCNPX et le fantôme approprié (fantôme 
anthropomorphe, parallélépipède et sphère CIUR). La distance entre la boîte à gants et le tronc du fantôme anthropomorphe est de l'ordre de $10 \mathrm{~cm}$. Les positions des fantômes des grandeurs opérationnelles sont plus délicates à définir. Cependant, deux positionnements implicites se dessinent sur ce poste de travail particulier :

- le premier est de situer ces fantômes avec le centre de la cellule de calcul (à $1 \mathrm{~cm}$ de profondeur dans le fantôme) à la même hauteur qu'un dosimètre porté au niveau de la poitrine côté cœur : soit à $140 \mathrm{~cm}$ de hauteur et à une position décalée de $15 \mathrm{~cm}$ par rapport à la boîte à gants $(10 \mathrm{~cm}$ de décalage latéral par rapport au centre du fantôme IRSN),

- le second est de positionner la cellule de calcul du fantôme à la hauteur de la source.

On s'attend donc à obtenir une valeur plus importante dans le second cas, à distance équivalent de la boîte à gants, puisque le fantôme est plus près de la source.

Les résultats correspondants obtenus et les positionnements des fantômes sont résumés dans le tableau I.

Alors que trois simulations ont suffi pour estimer les équivalents de dose, le calcul de la dose efficace $E$ a nécessité une quarantaine de simulations du fait de

TABLEAU I

Résultats des calculs avec les fantômes.

Results of the calculations with phantoms.

\begin{tabular}{ccccc}
\hline $\mathbf{N}^{\circ}$ & Grandeur & Positions du centre du fantôme & Résultat $(\mu \mathbf{S v} / \mathbf{h})$ & Remarques \\
\hline 1 & $E$ & $10 \mathrm{~cm} /$ boîte à gants & 31 & Fonction continue $w_{R}$ \\
2 & $E$ & $10 \mathrm{~cm} /$ boîte à gants & 26 & Paliers $w_{R}$ \\
3 & $H_{p}(10)$ & $\begin{array}{c}22,5 \mathrm{~cm} / \text { boîte à gants } \\
10 \mathrm{~cm} \text { à gauche du milieu du tronc }\end{array}$ & 100 & Coefficients de Siebert
\end{tabular}

$$
150 \mathrm{~cm} / \mathrm{sol}
$$

\begin{tabular}{|c|c|c|c|c|}
\hline 4 & $H^{*}(10)$ & $\begin{array}{c}30 \mathrm{~cm} / \text { boîte à gants } \\
10 \mathrm{~cm} \text { à gauche du milieu du tronc } \\
150 \mathrm{~cm} / \mathrm{sol}\end{array}$ & 89 & Coefficients de Siebert \\
\hline 5 & $H_{p}(10)$ & $\begin{array}{c}17,5 \mathrm{~cm} / \text { boîte à gants } \\
0 \mathrm{~cm} / \mathrm{milieu} \text { du tronc } \\
138 \mathrm{~cm} / \mathrm{sol}\end{array}$ & 228 & Coefficients de Siebert \\
\hline 6 & $H^{*}(10)$ & $\begin{array}{c}25 \mathrm{~cm} / \text { boîte à gants } \\
0 \mathrm{~cm} / \text { milieu du tronc } \\
138 \mathrm{~cm} / \mathrm{sol}\end{array}$ & 201 & Coefficients de Siebert \\
\hline 7 & $H_{p}(10)$ & $\begin{array}{c}30,5 \mathrm{~cm} / \text { boîte à gants } \\
0 \mathrm{~cm} / \mathrm{milieu} \text { du tronc } \\
130 \mathrm{~cm} / \mathrm{sol}\end{array}$ & 106 & Coefficients de Siebert \\
\hline 8 & $H^{*}(10)$ & $\begin{array}{c}38 \mathrm{~cm} / \text { boîte à gants } \\
0 \mathrm{~cm} / \text { milieu du tronc } \\
130 \mathrm{~cm} / \mathrm{sol}\end{array}$ & 103 & Coefficients de Siebert \\
\hline
\end{tabular}


la dépendance du facteur de pondération $w_{R}$ en fonction de l'énergie des neutrons et du type de rayonnements incidents. En effet, les neutrons émis par la source interagissent avec le verre plombé de la boîte à gants et dans une moindre mesure avec le béton du sol et des murs environnants. Les distributions énergétiques et angulaires des neutrons émis par la source sont ainsi modifiées. De plus, des photons sont créés par les neutrons dans le verre au plomb. La carte historique PTRAC de MCNPX a permis de produire les sources secondaires de neutrons et de photons sortant de la boîte à gants. Le type de rayonnements, l'énergie, le poids statistique des particules et leur direction de vol ont été sauvegardés dans les fichiers de sortie ptrac. Le facteur de pondération des neutrons $w_{R}$ dépendant de l'énergie des neutrons ; la volumétrie des fichiers de sortie des fichiers PTRAC a été réduite en employant des filtres en énergie au niveau de l'enregistrement des particules. À chaque découpe énergétique correspond une source secondaire que MCNPX reconnaît et traite, une fois le fichier source.f modifié. Le code a été de ce fait compilé pour tenir compte de ce type de source. Une autre solution aurait été d'utiliser la carte PTRAC avec un filtre sur les tallies f6 mais cela n'a pas été exploité dans ces simulations.

Les équivalents de dose obtenus surestiment la dose efficace $E$ d'un facteur 3 lorsque le fantôme est positionné à la hauteur du dosimètre. On observe un facteur 6 lorsque la face d'entrée des 3 fantômes est à $10 \mathrm{~cm}$ de la boîte à gants, cependant comme les fantômes CIUR ont leur partie supérieure exposée au niveau du combustible du pot, la distance entre la source et le fantôme est inférieure à celle observée à $150 \mathrm{~cm}$ du sol. La distance source-fantôme est prépondérante comme l'illustrent les calculs à $130 \mathrm{~cm}$ du sol.

Le fait de ne pas observer de sous-estimation de la dose efficace $E$ avec les grandeurs opérationnelles est plutôt satisfaisant du point de vue de l'estimation du risque radiologique encouru par le travailleur, toutefois la surestimation observée est trop importante. L'écart important observé entre les grandeurs s'explique par le fait que les organes les plus radiosensibles dans cette exposition sont plus éloignées de la source que les fantômes CIUR utilisées pour le calcul.

$\mathrm{Au}$ vu de ses résultats, la question que l'on peut se poser, hormis le positionnement des fantômes CIUR, est la suivante : Quelle est l'influence des distributions hétérogènes sur le résultat des grandeurs opérationnelles ?

\section{2. Évaluation des grandeurs en utilisant les coefficients de conversion ( $2^{e}$ méthode)}

Le code 1D déterministe SAS1 (2000) a été utilisé pour cette configuration assez simple à modéliser, cependant seul un « équivalent de dose d'ambiance » avec des 


\section{TABLEAU II}

Comparaison de résultats de SAS1 et MCNPX. Comparison of results obtained using SAS1 and MCNPX.

\begin{tabular}{|c|c|c|c|}
\hline Grandeur & Positionnement & Résultat $(\mu \mathrm{Sv} / \mathbf{h})$ & Remarques \\
\hline$H^{*}(10)$ SAS 1 & $\begin{array}{c}23 \mathrm{~cm} \text { boîte à gants } \\
0 \mathrm{~cm} / \text { milieu du tronc } \\
138 \mathrm{~cm} / \mathrm{sol}\end{array}$ & 66 & $\begin{array}{c}\text { Modélisation sphérique } \\
\text { ANS 6.1.1 }\end{array}$ \\
\hline$H^{*}(10) \mathrm{MCNPX}$ & $\begin{array}{c}23 \mathrm{~cm} \text { boîte à gants } \\
0 \mathrm{~cm} / \text { milieu du tronc } \\
138 \mathrm{~cm} / \mathrm{sol}\end{array}$ & 72 & $\begin{array}{c}\text { Sphère } 0,5 \mathrm{~cm} \text { tally } 5 \\
\text { ANS } 6.1 .1\end{array}$ \\
\hline
\end{tabular}

TABLEAU III

Résultats des calculs MCNPX de $H^{*}(10)$.

MCNPX results of $H^{*}(10)$ calculations.

\begin{tabular}{|c|c|c|c|}
\hline $\mathbf{N}^{\circ}$ & Grandeur & Centre de la sphère de $0,5 \mathrm{~cm}$ de $\mathrm{f5}$ & Résultat $(\mu \mathrm{Sv} / \mathrm{h})$ \\
\hline 1 & $H^{*}(10)$ & $\begin{array}{l}10 \mathrm{~cm} / \text { boîte à gants, } 0 \mathrm{~cm} / \text { milieu du tronc } \\
130 \mathrm{~cm} / \mathrm{sol}\end{array}$ & 217 \\
\hline 2 & $H^{*}(10)$ & $\begin{array}{c}10 \mathrm{~cm} / \text { boîte à gants, } 0 \mathrm{~cm} / \text { milieu du tronc } \\
138 \mathrm{~cm} / \mathrm{sol}\end{array}$ & 188 \\
\hline 3 & $H^{*}(10)$ & $\begin{array}{l}10 \mathrm{~cm} / \text { boîte à gants, } 0 \mathrm{~cm} / \text { milieu du tronc } \\
\qquad 150 \mathrm{~cm} / \mathrm{sol}\end{array}$ & 105 \\
\hline 4 & $H^{*}(10)$ & $\begin{array}{l}15 \mathrm{~cm} / \text { boîte à gants, } 10 \mathrm{~cm} \text { à gauche du milieu du tronc } \\
130 \mathrm{~cm} / \mathrm{sol}\end{array}$ & 121 \\
\hline 5 & $H^{*}(10)$ & $\begin{array}{l}15 \mathrm{~cm} / \text { boîte à gants, } 10 \mathrm{~cm} \text { à gauche du milieu du tronc } \\
138 \mathrm{~cm} / \mathrm{sol}\end{array}$ & 112 \\
\hline 6 & $H^{*}(10)$ & $\begin{array}{l}15 \mathrm{~cm} / \text { boîte à gants, } 0 \mathrm{~cm} \text { à gauche du milieu du tronc } \\
150 \mathrm{~cm} / \mathrm{sol}\end{array}$ & 77 \\
\hline 7 & $H^{*}(10)$ & $\begin{array}{l}23 \mathrm{~cm} / \text { boîte à gants, } 0 \mathrm{~cm} / \text { milieu du tronc } \\
130 \mathrm{~cm} / \mathrm{sol}\end{array}$ & 87 \\
\hline 8 & $H^{*}(10)$ & $\begin{array}{l}23 \mathrm{~cm} / \text { boîte à gants, } 0 \mathrm{~cm} / \text { milieu du tronc } \\
138 \mathrm{~cm} / \mathrm{sol}\end{array}$ & 83 \\
\hline 9 & $H^{*}(10)$ & $\begin{array}{c}23 \mathrm{~cm} / \text { boîte à gants, } 0 \mathrm{~cm} / \text { milieu du tronc } \\
150 \mathrm{~cm} / \mathrm{sol}\end{array}$ & 63 \\
\hline
\end{tabular}

coefficients issus de la publication ANSI/ANS-6.1.1 (ANSI, 1977) peut être estimé. Le tableau II présente les résultats obtenus avec ce code et MCNPX. On observe un bon accord entre les deux codes.

Des calculs avec MCNPX et les coefficients de conversion de la CIUR 57 ont également été réalisés ( $c f$. Tab. III).

Les résultats du tableau III illustrent le même phénomène que précédemment, c'est-à-dire l'éloignement de la source conduit à une diminution de la dose. 
Poursuivre l'étude à des positions plus éloignées de l'axe médian du pot et effectuer la moyenne des équivalents de dose obtenus ainsi permettraient de se rapprocher de la dose efficace $E$ évaluée pour ce poste.

Les groupes de résultats suivants sont du même ordre de grandeur :

- résultat 4 du tableau I et résultat 6 du tableau III,

- résultat 6 du tableau I et résultat 2 du tableau III,

- résultat 8 du tableau I et résultat 7 du tableau III.

Les différences observées sont de l'ordre de $10 \%$. La variation des distributions spectrales de la fluence en fonction des positions étudiées rend délicate l'interprétation des résultats obtenus avec les deux méthodes. En effet, on s'écarte quelque peu du champ expansé unidirectionnel auquel est exposée la sphère CIUR.

\section{Conclusion}

Le schéma de calcul de la dose efficace retenu par l'IRSN (fantôme + code Monte Carlo) permet d'évaluer la grandeur de protection à mieux que $10 \%$ pour les configurations d'irradiation homogène et monoénergétique de (ICRP, 1990; ICRU, 1998).

L'étude dosimétrique d'un poste de travail auprès d'une boîte à gants a montré la forte dépendance des résultats par rapport aux positionnements des fantômes et des points de calcul. Une étude expérimentale de caractérisation dosimétrique de ce poste de travail conduirait à une dépendance identique par rapport au positionnement des appareils de mesures.

Aucune sous-estimation de la dose efficace $E$ par les grandeurs opérationnelles n'a été observée quelle que soit la méthode de calcul retenue. Cependant les écarts obtenus entre les estimateurs et la dose efficace ne sont pas satisfaisants du fait de leur importance.

Conclure de cette étude que l'objectif d'estimation de la dose efficace $E$ à l'aide des grandeurs opérationnelles n'est pas atteint pour des champs hétérogènes de rayonnements neutroniques et photoniques serait prématuré. Seule une étude géométrique plus systématique des dépendances des résultats et l'évaluation dosimétrique d'autres postes de travail permettraient d'infirmer ou de confirmer ce point.

L'objectif premier de ce travail était de démontrer l'intérêt de disposer d'un outil de mesure de la dose efficace $E$ au lieu d'instruments évaluant les grandeurs opérationnelles en champs neutroniques ; ceci afin de s'assurer que les dosimètres 
individuels portés par les travailleurs sont adaptés aux conditions d'exposition radiologique des ces derniers. Cet objectif a été atteint pour ce poste mais le travail est à poursuivre pour des postes présentant des hétérogénéités différentes.

Les travaux de thèse de Furstoss (2006) ayant montré la plus value d'un tel instrument en champs photoniques, le développement d'un fantôme instrumenté est en cours à l'IRSN.

\section{RÉFÉRENCES}

ANSI (1977) American National Standard Neutron and Gamma-Ray Flux-to-Dose Rate Factors, ANSI/ANS-6.1.1-1977 (N666), American Nuclear Society.

Cristy M., Eckerman K.F. (1987) Specific absorbed fractions of energy at various ages from internal photon sources, I. Methods, Report Health and Safety Research Division of Oak Ridge National Laboratory, TM-8381/V1.

Furstoss C. (2006) Mémoire de doctorat es Sciences de l'Université Paris XI, soutenu le 20/11/2006, Conception et développement d'un fantôme anthropomorphe équipé de détecteurs dans le but d'évaluer la dose efficace au poste de travail : étude de faisabilité.

Furstoss C., Ménard S. (2005) Description de la géométrie du fantôme mathématique anthropomorphe utilisé pour la modélisation avec les codes Monte Carlo MCNP et MCNPX, Rapport IRSN DRPH/SDE $n^{\circ}$ 2005-13.

Furstoss C., Ménard S., Clairand I., Chau Q. (2007) A phantom for effective dose measurement: organ dose distribution assessment based on a numerical approach, Radiat. Prot. Dosim. DOI: $10.1093 / \mathrm{rpd} / \mathrm{ncm} 186$.

Hendricks J.S., Mc Kinney G.W., Waters L.S. (2005) MCNPX Version 2.5.0, User's Manual LA-CP05-0369

ICRP Publication 60 (1990) Recommendations of the International Commission on Radiological Protection, Ann. ICRP 21(1-3).

ICRU Report 57 (1998) Conversion Coefficients for use in Radiological Protection Against External Radiation.

Kramer R., Zankl M., Williams G., Drexler G. (1982) The calculation of dose from external photon exposures using reference human phantoms and Monte Carlo methods, Part I: the male (ADAM) and female (EVA) adult mathematical phantom. GSF-Bericht S-885.

SAS1 (2000) A one-dimensional shielding analysis module, NUREG/CR-0200 Revision 6, Volume 1, Section S1, ORNL/NUREG/CSD-2/V1/R6.

Siebert B.R.L., Schumacher H. (1992) Quality factors and ambient dose equivalent for neutrons based on the new ICRP recommendations, Radiat. Prot. Dosim. 40, 85-89. 